Supporting Information

\title{
Synthesis of Aryl Ketones by the Pd-Catalyzed C-H Activation of Arenes and Intermolecular Carbopalladation of Nitriles
}

\author{
Chengxiang Zhou and Richard C. Larock* \\ Department of Chemistry, Iowa State University, Ames, Iowa 50011
}

larock@iastate.edu

\section{General}

The ${ }^{1} \mathrm{H}$ and ${ }^{13} \mathrm{C}$ NMR spectra were recorded at 300 or 400 and 75 or $100 \mathrm{MHz}$, respectively. Thin layer chromatography was performed using commercially prepared 60mesh silica gel plates (Whatman K6F), and visualization was effected with short wavelength UV light (254 nm). Low-resolution mass spectra were recorded on a Finnigan TSQ700 triple quadruple mass spectrometer (Finnigan MAT, San Jose, CA). Highresolution mass spectra were recorded on a Kratos MS50TC double focusing magnetic sector mass spectrometer using EI at $70 \mathrm{eV}$. The arenes and nitriles were purchased from Sigma-Aldrich Co. and were used without further purification. All palladium salts were donated by Johnson Matthey Inc. and Kawaken Fine Chemicals Co., Ltd. All boronic acids were donated by Frontier Scientific Co., Ltd.

\section{The intermolecular reaction of nitriles and arenes (Table 2, entries 1 - 8)}

The arene $(2.0 \mathrm{mmol})$, the nitrile $(1.0 \mathrm{mmol}), \mathrm{Pd}(\mathrm{OAc})_{2}(0.10 \mathrm{mmol})$, DMSO $(0.10$ $\mathrm{mL})$ and TFA $(2.5 \mathrm{~mL})$ were placed in a 6 dram vial. The vial was sealed and the contents were stirred and heated at the indicated temperature for $24 \mathrm{~h}$. The ketone products were obtained using the following acid work-up procedure and the ketimine products were obtained by using the following basic work-up procedure.

Acidic work-up. Water $(15 \mathrm{~mL})$ was added to the vial and the resulting mixture was heated at $70{ }^{\circ} \mathrm{C}$ for $2 \mathrm{~h}$. The mixture was then cooled and extracted with diethyl ether three times. The combined organic layers were dried over anhydrous $\mathrm{MgSO}_{4}$ and the solvent was evaporated under reduced pressure. The crude product was purified by chromatography on a silica gel column.

Basic Work-up. The reaction mixture was cooled to room temperature, and then $\mathrm{K}_{2} \mathrm{CO}_{3}$ was added to the vial until no $\mathrm{CO}_{2}$ bubbles were generated (Caution: a large amount of $\mathrm{CO}_{2}$ is generated due to the reaction of TFA and $\mathrm{K}_{2} \mathrm{CO}_{3}$ ). The resulting mixture was extracted with diethyl ether three times. The combined organic layers were dried over anhydrous $\mathrm{MgSO}_{4}$ and the solvent was evaporated under reduced pressure. The crude product was purified by chromatography on a silica gel column.

\section{The intramolecular reaction of arylalkanenitriles (Table 2, entries 9 - 10)}

The nitrile $(0.2 \mathrm{mmol}), \mathrm{Pd}(\mathrm{OAc})_{2}(0.03 \mathrm{mmol}), \mathrm{DMSO}(0.2 \mathrm{~mL})$ and TFA $(5.0 \mathrm{~mL})$ were placed in a 6 dram vial. The vial was sealed and the contents were stirred and heated at the indicated temperature for $24 \mathrm{~h}$. The resulted mixture was then worked up according to the above acidic work-up procedure. 


\section{The intermolecular reaction of nitriles and boronic acids (Scheme 2)}

The nitrile $(5.0 \mathrm{mmol})$, the boronic acid $(1.0 \mathrm{mmol}), \mathrm{Pd}(\mathrm{OAc})_{2}(0.10 \mathrm{mmol}), \mathrm{DMSO}$ $(0.10 \mathrm{~mL})$ and TFA $(2.5 \mathrm{~mL})$ were placed in a 6 dram vial. The vial was sealed and the contents were stirred and heated at $90{ }^{\circ} \mathrm{C}$ for $24 \mathrm{~h}$. The resulted mixture was then worked up according to the above acidic or basic work-up procedure.

\section{Characterization data:}<smiles>Cc1cc(C)c(C(=N)c2ccccc2)c(C)c1</smiles>

2,4,6-Trimethylbenzophenone imine. ${ }^{1} \quad$ Light yellow oil; ${ }^{1} \mathrm{H}$ NMR (300 $\mathrm{MHz}$, $\left.\mathrm{CDCl}_{3}\right) \delta 2.10(\mathrm{~s}, 6 \mathrm{H}), 2.34(\mathrm{~s}, 3 \mathrm{H}), 6.92(\mathrm{~s}, 2 \mathrm{H}), 7.35-7.45(\mathrm{~m}, 3 \mathrm{H}), 7.73(\mathrm{dd}, J=8.1,1.8$ $\mathrm{Hz}, 2 \mathrm{H}), 8.80-9.70(\mathrm{~s}, 1 \mathrm{H}) ;{ }^{13} \mathrm{C} \mathrm{NMR}\left(75 \mathrm{MHz}, \mathrm{CDCl}_{3}\right) \delta 19.85,21.35,128.00,128.60$, $128.85,131.42,134.58,137.64,137.75,138.15,179.05 ;$ IR $\left(\mathrm{CDCl}_{3}, \mathrm{~cm}^{-1}\right) 3265,3063$, 2925, 2866, 1606, 1573, 1469, and 1448; MS (EI) m/z (rel intensity) $223\left(\mathrm{M}^{+}, 22\right), 22$ (100), 207 (40); HRMS (EI) calcd for $\mathrm{C}_{16} \mathrm{H}_{17} \mathrm{~N} 223.1361$, found: 223.1367.<smiles>COc1ccc(C(=N)c2c(C)cc(C)cc2C)cc1</smiles>

2,4,6-Trimethylphenyl-4'-methoxyphenylmethanimine. Yellow oil; ${ }^{1} \mathrm{H}$ NMR $\left(300 \mathrm{MHz}, \mathrm{CDCl}_{3}\right) \delta 2.09(\mathrm{~s}, 6 \mathrm{H}), 2.33(\mathrm{~s}, 3 \mathrm{H}), 3.83(\mathrm{~s}, 3 \mathrm{H}), 6.86-6.91(\mathrm{~m}, 4 \mathrm{H}), 7.66(\mathrm{~d}, J$ $=8.7 \mathrm{~Hz}, 2 \mathrm{H}), 8.0-9.2(\mathrm{~s}, 1 \mathrm{H}) ;{ }^{13} \mathrm{C} \mathrm{NMR}\left(75 \mathrm{MHz}, \mathrm{CDCl}_{3}\right) \delta 19.76,21.31,55.54,114.05$, 128.51, 129.64, 130.66, 134.46, 137.84, 138.36, 162.16, 178.02; IR $\left(\mathrm{CDCl}_{3}, \mathrm{~cm}^{-1}\right) 3260$, 3005, 2926, 2854, 1596, 1569, 1510, and 1460; MS (EI) m/z (rel intensity) $253\left(\mathrm{M}^{+}, 28\right)$, 252 (100), 237 (22); HRMS (EI) calcd for $\mathrm{C}_{17} \mathrm{H}_{19} \mathrm{NO} 253.1467$, found: 253.1471.<smiles>Cc1cc(C)c(C(=N)c2ccc(Br)cc2)c(C)c1</smiles>

2,4,6-Trimethylphenyl-4'-bromophenylmethanimine. Light red oil; ${ }^{1} \mathrm{H}$ NMR (300 $\left.\mathrm{MHz}, \mathrm{CDCl}_{3}\right) \delta 2.07(\mathrm{~s}, 6 \mathrm{H}), 2.32(\mathrm{~s}, 3 \mathrm{H}), 6.91(\mathrm{~s}, 2 \mathrm{H}), 7.48(\mathrm{~d}, J=8.7 \mathrm{~Hz}, 2 \mathrm{H}), 7.59$ (d, $J=8.7 \mathrm{~Hz}, 2 \mathrm{H}), 8.8-10.2(\mathrm{~s}, 1 \mathrm{H}) ;{ }^{13} \mathrm{C} \mathrm{NMR}\left(75 \mathrm{MHz}, \mathrm{CDCl}_{3}\right) \delta 19.80,21.34,126.00$, $128.68,129.52,132.02,134.42,136.91,137.53,138.26,177.77 ;$ IR $\left(\mathrm{CDCl}_{3}, \mathrm{~cm}^{-1}\right) 3254$, 3027, 2920, 2858, 1611, 1586, 1561, and 1483; MS (EI) m/z (rel intensity) 302 (100), 300 (100), 287 (20), 285 (21), 222 (35); HRMS (EI) calcd for $\mathrm{C}_{16} \mathrm{H}_{16} \mathrm{BrN} 301.0466$, found: 301.0473 . 


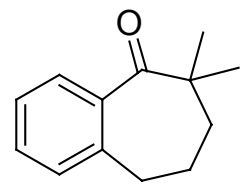

6,6-Dimethyl-6,7,8,9-tetrahydrobenzocyclohepten-5-one. ${ }^{2} \quad{ }^{1} \mathrm{H}$ NMR (400 MHz, $\left.\mathrm{CDCl}_{3}\right) \delta 1.17(\mathrm{~s}, 6 \mathrm{H}), 1.66(\mathrm{t}, J=6.8 \mathrm{~Hz}, 2 \mathrm{H}), 1.89(\mathrm{~m}, 2 \mathrm{H}), 2.76(\mathrm{t}, J=6.8 \mathrm{~Hz}, 2 \mathrm{H})$, $7.10(\mathrm{~d}, J=7.6 \mathrm{~Hz}, 1 \mathrm{H}), 7.24-7.26(\mathrm{~m}, 2 \mathrm{H}), 7.32-7.35(\mathrm{~m}, 1 \mathrm{H}) ;{ }^{13} \mathrm{C} \mathrm{NMR}(75 \mathrm{MHz}$, $\left.\mathrm{CDCl}_{3}\right) \delta 23.38,25.93,31.16,37.73,46.17,126.72,127.19,128.69,130.89,137.47$, 141.50, 215.33; IR $\left(\mathrm{CDCl}_{3}, \mathrm{~cm}^{-1}\right) 3023,2935,2865,1679,1599,1472$, and 1453; MS (EI) $\mathrm{m} / \mathrm{z}$ (rel intensity) $188\left(\mathrm{M}^{+}, 14\right), 130(85), 120$ (100); HRMS (EI) calcd for $\mathrm{C}_{13} \mathrm{H}_{16} \mathrm{O}$ 188.1201, found: 188.1205 .

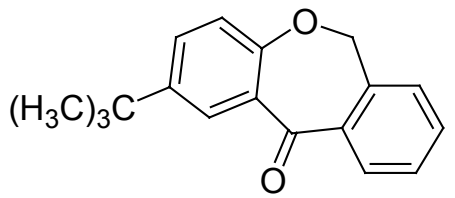

2-(tert-Butyl)dibenz[b,e]oxepin-11(6H)-one. Colorless oil; ${ }^{1} \mathrm{H}$ NMR $(400 \mathrm{MHz}$, $\left.\mathrm{CDCl}_{3}\right) \delta 1.35(\mathrm{~s}, 9 \mathrm{H}), 5.17(\mathrm{~s}, 2 \mathrm{H}), 6.99(\mathrm{~d}, J=8.4 \mathrm{~Hz}, 2 \mathrm{H}), 7.35(\mathrm{~d}, J=7.6 \mathrm{~Hz}, 1 \mathrm{H})$, 7.45-7.48 (m, 1H), 7.53-7.56 (m, 3H), 7.89 (d, $J=8.4 \mathrm{~Hz}, 1 \mathrm{H}), 8.23(\mathrm{~s}, 1 \mathrm{H}) ;{ }^{13} \mathrm{C} \mathrm{NMR}$ $\left(75 \mathrm{MHz}, \mathrm{CDCl}_{3}\right) \delta 31.56,34.63,73.76,120.52,124.79,127.93,128.17,129.39,129.65$, 132.81, 133.35, 135.86, 140.96, 145.05, 159.48, 191.71; IR $\left(\mathrm{CDCl}_{3}, \mathrm{~cm}^{-1}\right) 3032,2963$, 2868, 1642, 1605, 1490, and 1463; MS (EI) m/z (rel intensity) $266\left(\mathrm{M}^{+}, 29\right), 251$ (100); HRMS (EI) calcd for $\mathrm{C}_{18} \mathrm{H}_{18} \mathrm{O}_{2} 266.1307$, found: 266.1311 .

\section{References:}

1) Luo, F. H.; Chu, C. I.; Cheng, C. H. Organometallics 1998, 17, 1025.

2) Dunkelblum, E.; Hart, H.; Suzuki, M. J. Am. Chem. Soc. 1977, 99, 5074. 

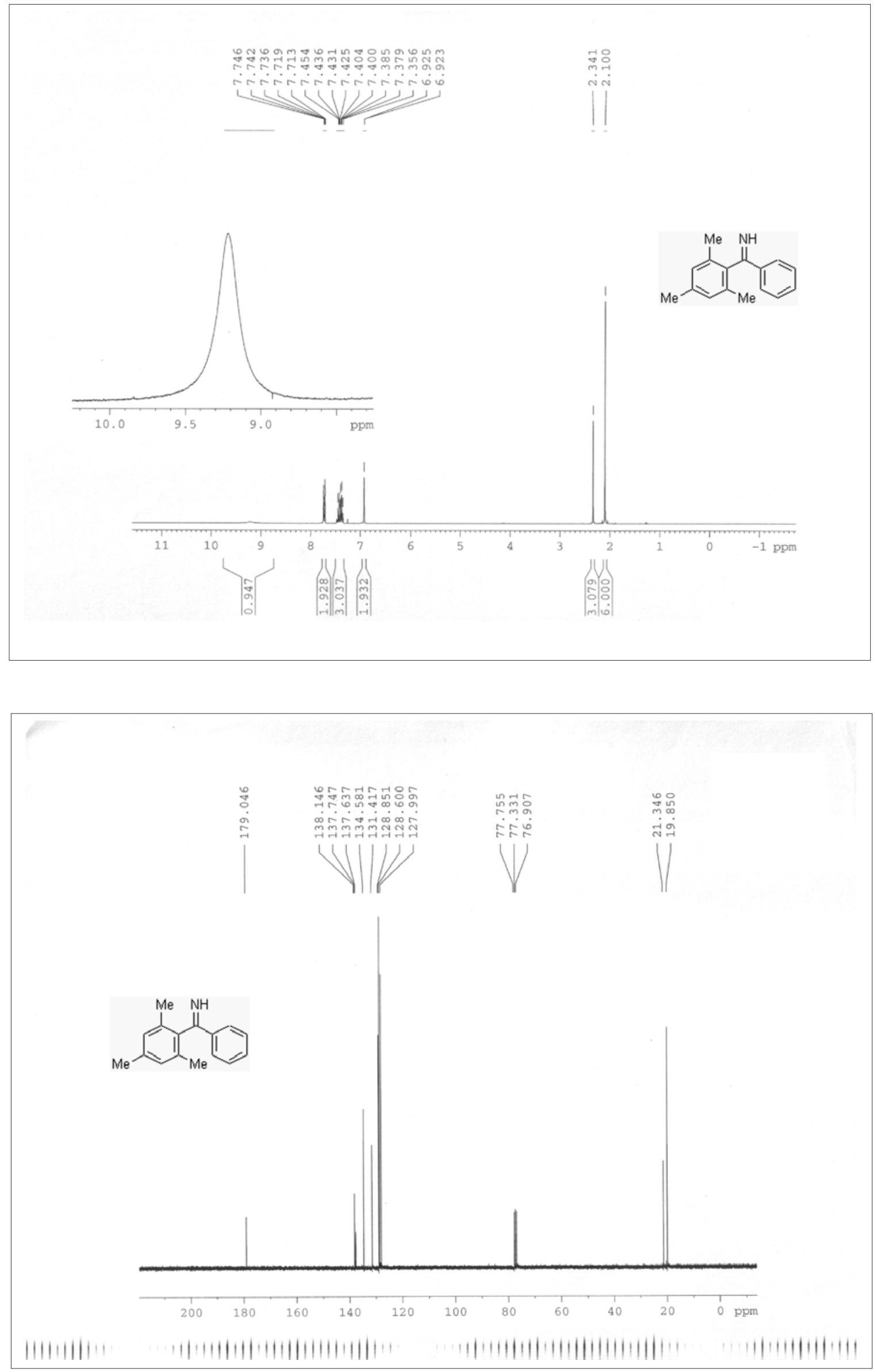

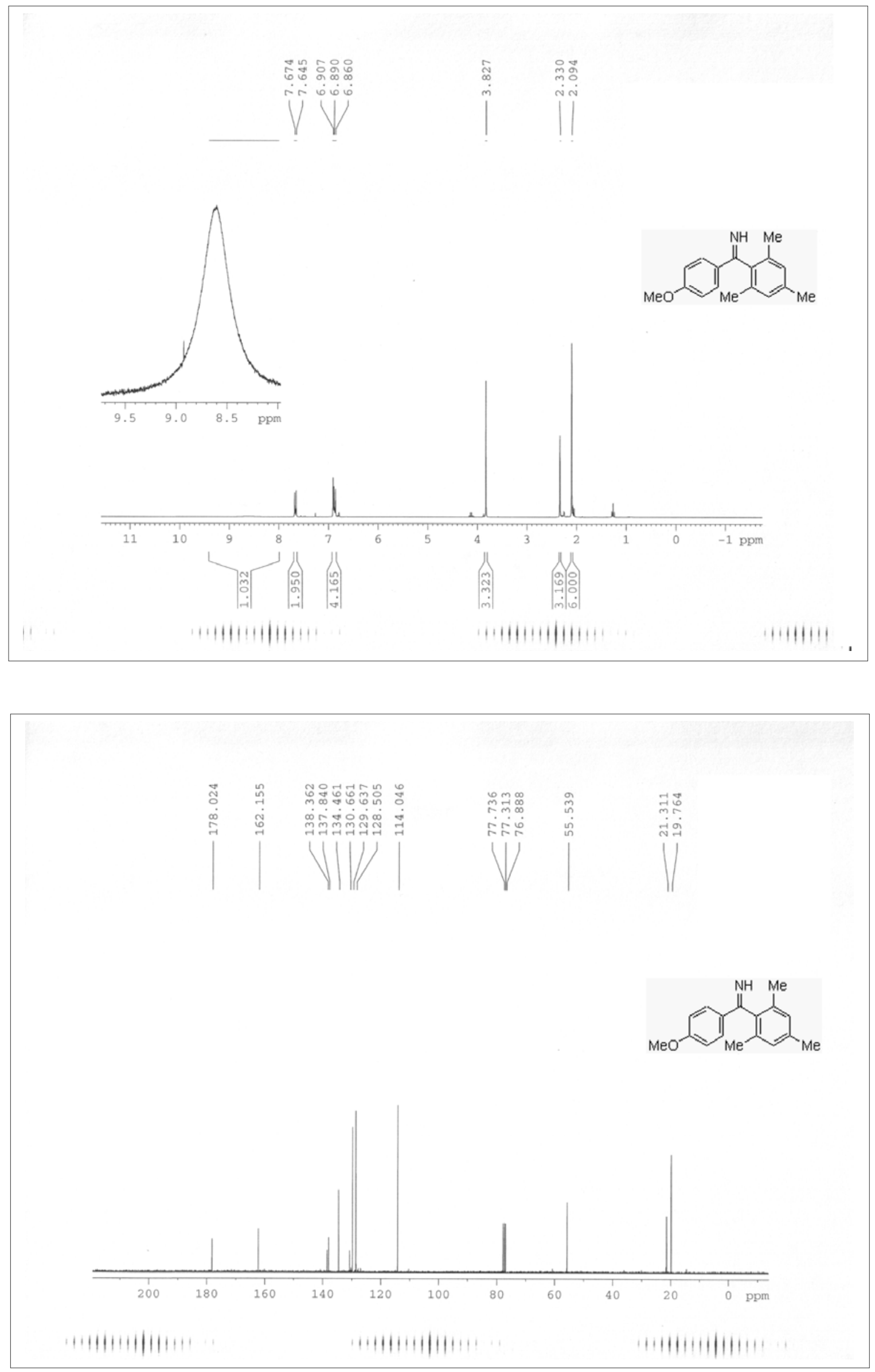

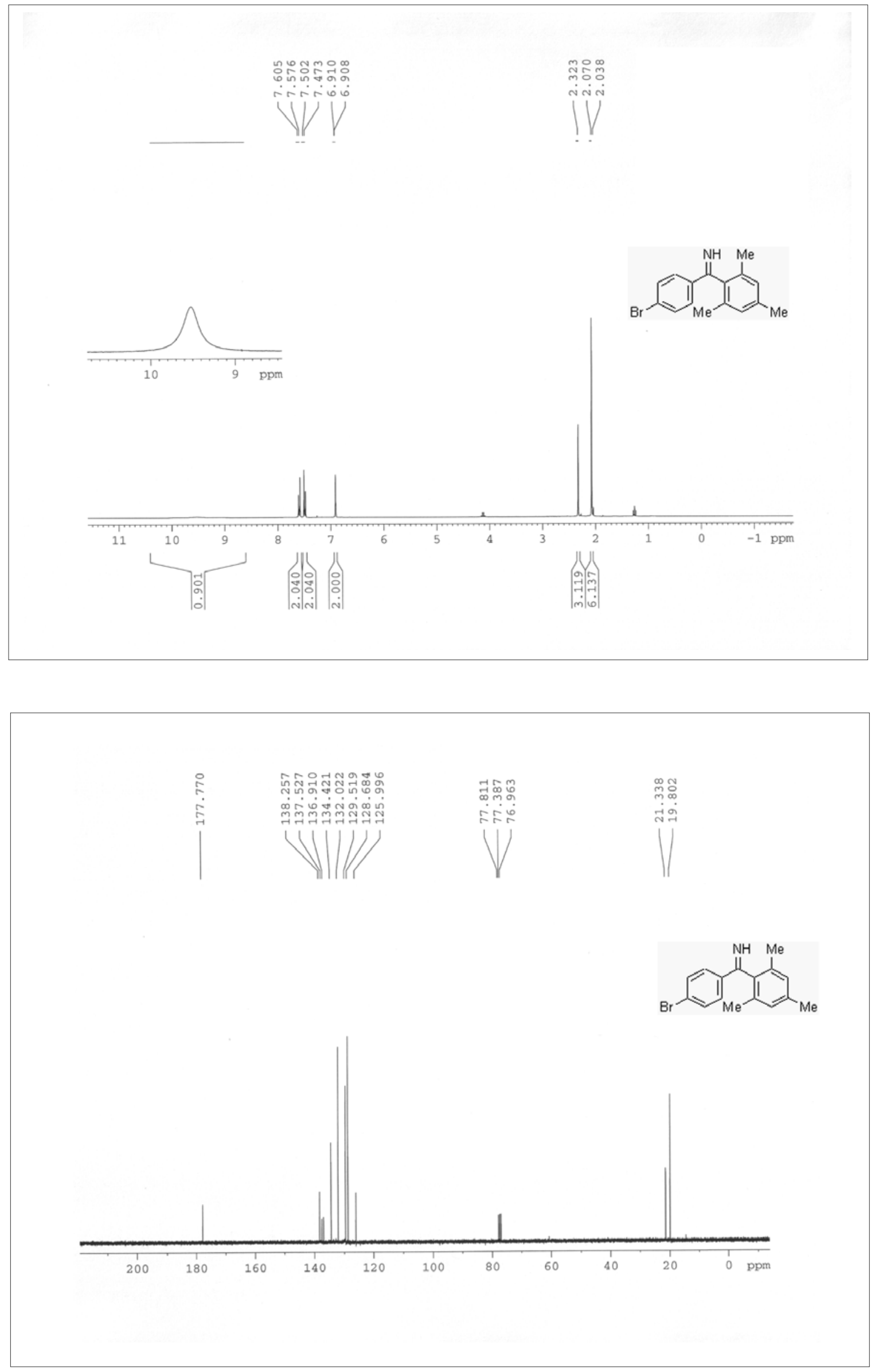

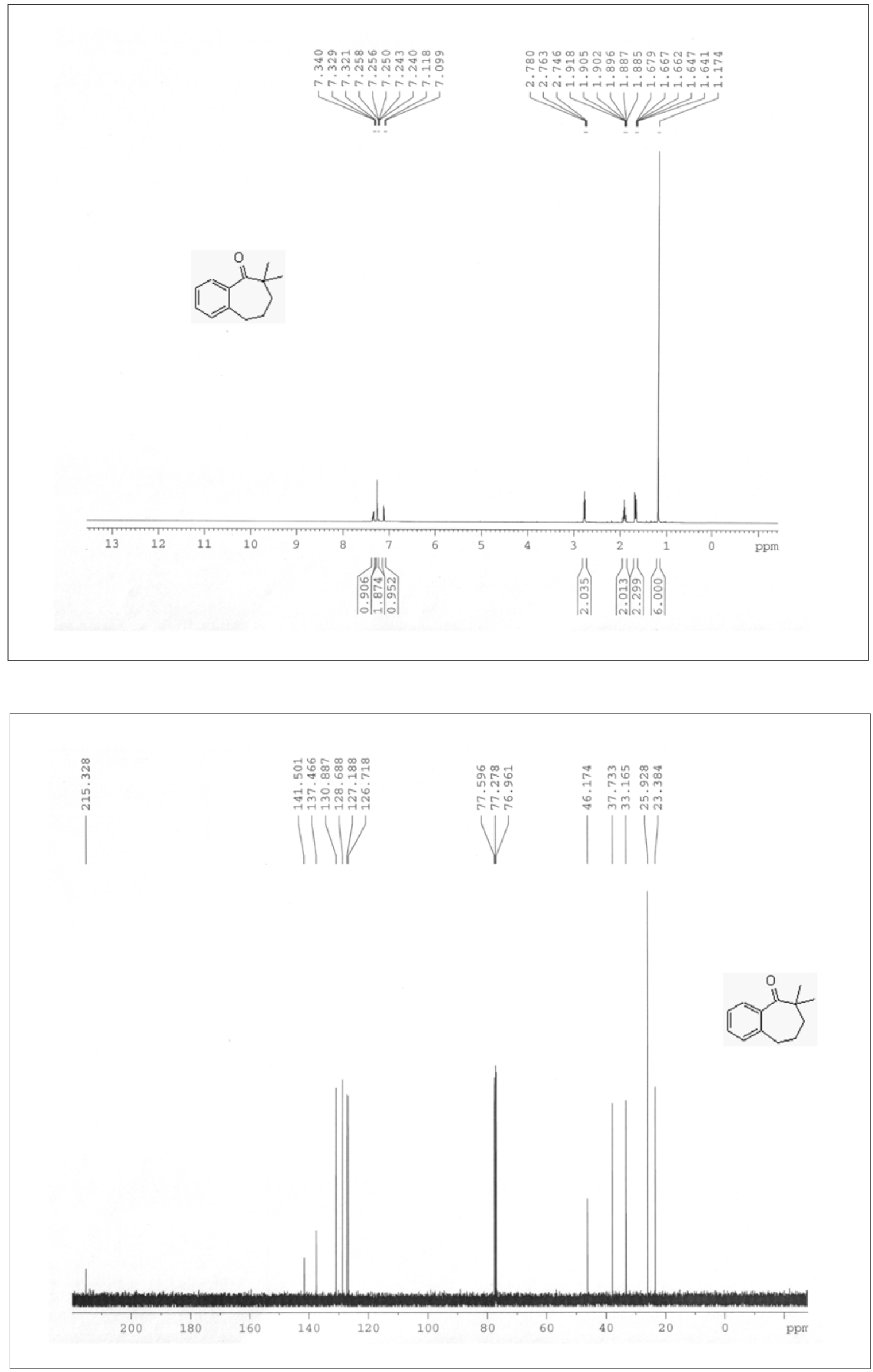

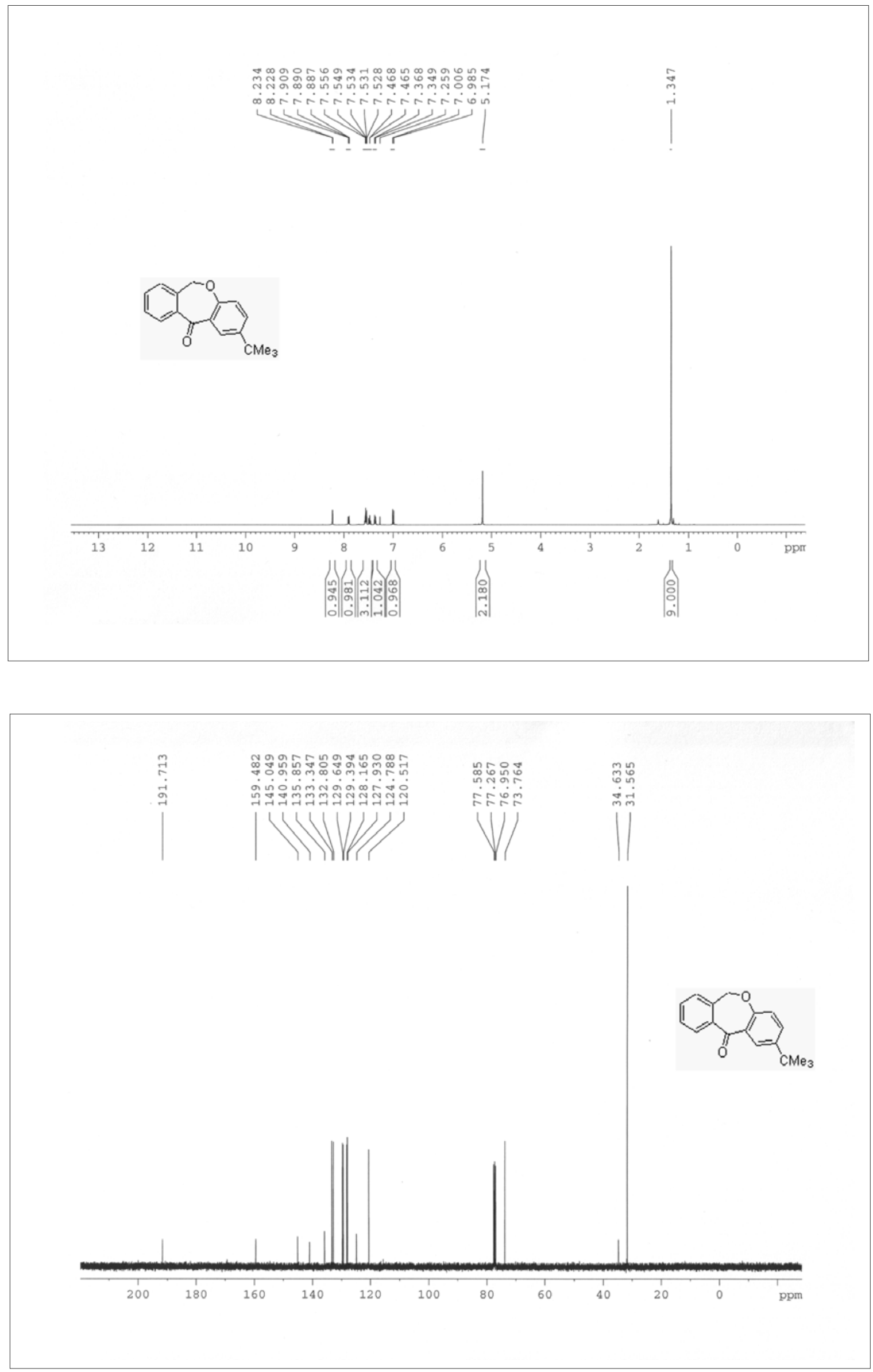